\title{
MANGANÊS E GERMINAÇÃO DE ESPOROS DE FUNGOS MICORRÍZICOS ARBUSCULARES IN VITRO ${ }^{(1)}$
}

\author{
E. J . B. N. CARDOSO(2), R. B. NAVARRO(3) \& M. A. NOGUEIRA(4)
}

\begin{abstract}
RESUMO
A alta disponibilidade de íons metálicos no solo, dentre eles o $\mathrm{Mn}^{2+}$, pode ini bir os fungos micorrízicos arbusculares (FMA), retardando a germinação dos esporos e, conseqüentemente, a formação de micorriza, o que reduz a eficiência simbiótica. O objetivo deste trabalho foi avaliar o efeito do íon $\mathrm{Mn}^{2+}$ sobre a germi nação de esporos de seis espécies de FMA dos gêneros Glomus, Acaulospora, Gigaspora e Scutel lospora em experimento in vitro. Em substrato constituído

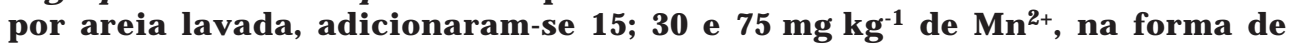
$\mathrm{MnCl}_{2} \cdot \mathbf{4} \mathrm{H}_{2} \mathrm{O}$, mantendo-se um controle sem adição de $\mathrm{Mn}$. Acondicionaram-se os esporos em envelopes membranosos, introduzindo-os entre duas camadas de areia com diferentes níveis de Mn, em placas de Petri. Após 30 dias de incubação, avaliou-se a germi nação dos esporos. Houve decrésci mo médio de 32, 49 e 75 \% na germinação dos esporos, à medida que se aumentaram as doses de $\mathrm{Mn}$, em comparação ao controle. A germinação de esporos do gênero Glomus foi totalmente inibida na maior dose. $O$ gênero Acaul ospora sofreu decréscimos de até $50 \%$ já na dose de $15 \mathrm{mg} \mathrm{kg}^{-1} \mathrm{de}_{\mathrm{Mn}}{ }^{2+}$, enquanto os gêneros Scutel lospora e Gigaspora apresentaram os maiores índices de germinação de esporos, tendo havi do tolerância no caso de Gigaspora, mesmo na maior dose de $\mathrm{Mn}^{2+}$, e estímulo

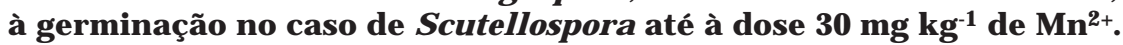

Termos de indexação: fungistase, metal pesado, Glomales.

(1) Recebido para publicação em setembro de 2001 e aprovado em março de 2002.

(2) Professora Titular do Departamento de Solos e Nutrição de Plantas da Escola Superior deAgricultura "Luiz de Queiroz" - ESALQ/ USP. Caixa Postal 09, CEP 13418-900 Piracicaba (SP). Bolsista do CNPq. E-mail: ejbncard@esalq.usp.br

(3) Mestre em Microbiologia Agrícola, ESALQ/USP. E-mail: rasangelanavarro@hotmail.com

(4) Doutorando em Solos e Nutrição de Plantas, ESALQ/USP. Bolsista da FAPESP. E-mail: nogueira@esalq.usp.br 


\title{
SUMMARY: MANGANESE AND SPORE GERMINATION OF ARBUSCULAR MYCORRHIZAL FUNGI IN VITRO
}

\begin{abstract}
Thehigh availability of metallicions in thesoil, including $\mathrm{Mn}^{2+}$, may inhibit arbuscular mycorrhizal fungi (AMF), slowing down sporegermination and, consequently, themycorrhiza formation, therefore decreasing the symbiotic effectiveness. The aim of this study was to evaluatethe effect of $\mathrm{Mn}^{2+}+$ ions on sporegermination in six AMF species of thegenera Glomus, Acaul ospora, Gigaspora and Scutell ospora under in vitro conditions. Different $\mathrm{Mn}^{2+}$ doses (15, 30 and $75 \mathrm{mg} \mathrm{kg}^{-1}$ ) as $\mathrm{MnCl}_{2} \cdot 4 \mathrm{H}_{2} \mathrm{O}$ were added to a washed sand substrate, keeping a control without $\mathrm{Mn}$ addition. Spores wereaccommodated in membranous envel opes which were placed between two sand layers treated with theabove $\mathrm{Mn}$ doses in Petri dishes. After a 30 day incubati on period, sporegermination was evaluated, presenting decreases $(32,49$ and $75 \%$ ) as $\mathrm{Mn}$ doses increased, compared to the control. Sporegermi nation in thegenus Glomus was completel y inhi bi ted at thetop dose, whilein theAcaulospora genus it dropped down to $50 \%$ even at the lowest dose $\left(15 \mathrm{mg} \mathrm{kg}^{-1}\right.$ of $\left.\mathrm{Mn}^{2+}\right)$. The genera Scutel lospora and Gigaspora presented highest germination indexes, out of which Gigaspora presented $\mathrm{Mn}$ tolerance, even at the highest $\mathrm{Mn}^{2+}$ dose. Scutellospora presented stimulus for germination up to doses of $30 \mathrm{mg} \mathrm{kg}^{-1}$ of $\mathrm{Mn}^{2+}$.
\end{abstract}

Index terms: fungistasis, heavy metal, Glomales.

\section{NTRODUÇÃO}

A avaliação da compatibilidade das interações entre hospedeiro, ambiente e fungos micorrízicos arbusculares (FMA) geralmente é feita pela determinação do número de esporos no solo, pela capacidade de germinação dos esporos e pela col onização radicular interna (Clark, 1997). Os FMA são encontrados nos mais variados ambientes, em solos com pH variando de 2,7 a 9,2 (Daft \& ElGiahmi, 1975; Bowen, 1980). Entretanto, o efeito de baixos valores de $\mathrm{pH}$ do solo sobre os FMA é difícil deser avaliado isoladamente, uma vez que este fator aumenta a disponibilidade de vários el ementos, como os íons metálicos $\mathrm{Al}, \mathrm{Cu}, \mathrm{Fe}, \mathrm{Mn}$ e Zn (Lambais \& Cardoso, 1988). Esses íons, por sua vez, influenciam tanto os FMA quanto seus hospedeiros.

A germinação dos esporos e o desenvolvimento inicial das hifas fúngicas podem ser diminuídos ou inibidos pela presença excessiva de metais, o que pode atrasar ou suprimir a formação de micorriza (Koomen et al., 1990). Os efeitos tóxicos do Mn ocorrem normal mente em combinação com os do Al em solos com pH inferior a 5,5, cujos materiais de origem são ricos em $\mathrm{Mn}$. Entretanto, em solos com $\mathrm{pH}$ superior a 5,5, o alumínio é precipitado quase que completamente, ao passo que o $\mathrm{Mn}$ ainda está disponível (Foy, 1984), podendo causar efeitos adversos, se em excesso.

Os diferentes ecotipos de F MA podem apresentar diferentes graus de tolerância a metais: os isolados de locais com alta contaminação, ou com níveis naturalmente altos, como o caso do $\mathrm{Mn}$ e $\mathrm{Al}$ em muitos solos brasileiros, tendem a ser mais tolerantes na mesma situação (Weissenhorn et al., 1993; 1994). Weissenhorn et al. (1994) observaram que a germinação dos esporos de FMA isolados de locais contaminados por $\mathrm{Zn}$ e $\mathrm{Cd}$ foi menos inibida por esses elementos em comparação ao observado para esporos provenientes de locais não contaminados. Outra constatação foi que o índice de tolerância ao metal não foi específico, ou seja, esporos provenientes de campos contaminados com Cd também foram mais tol erantes a Zn, evice-versa.

Os mecanismos de adaptação dos FMA a altas concentrações de metais não são conhecidos. Entretanto, considerando o seu ciclo relativamente longo e o grande número de núcl eos em seus esporos, essa maior tolerância a metais podeestar relacionada com a plasticidade fenotípica e não com a sel eção de genótipos tolerantes (Weissenhorn et al., 1994).

A maioria dos trabalhos na literatura relata os efeitos fungistáticos de íons metálicos como o Al, Cd, Zn eCu, mas não oMn, sobre esporos deFMA. Dessa forma, este trabalho teve como objetivo avaliar o efeito do $\mathrm{Mn}$ solúvel na germinação in vitro de esporos de seis espécies de FMA.

\section{MATERIAL E MÉTODOS}

O substrato utilizado para avaliar a germinação dos esporos de FMA foi constituído por areia $(<2 \mathrm{~mm})$ tratada por $24 \mathrm{~h}$ com $\mathrm{HCl}$ a $5 \%$. Após esse tratamento, o substrato foi lavado com água até à 
retirada doácido, conforme constatado pela medição dos valores de pH após cada lavagem. Considerou-se que o ácido havia sido removido quando foram obtidos valores de $\mathrm{pH}$ em torno de 5 . A areia lavada foi submetida à autoclavagem a $121^{\circ} \mathrm{C}$, por $2 \mathrm{~h}$, e, em seguida, analisada quanto à sua composição química para fins defertilidade, revelando os seguintes resultados: $\mathrm{pH}\left(\mathrm{CaCl}_{2}\right)=5 ; \mathrm{P}$ (resina) $=4,8 \mathrm{mg} \mathrm{dm}^{-3}$; $\mathrm{Ca}=1,5 \mathrm{mmol}_{\mathrm{c}} \mathrm{dm}^{-3} ; \mathrm{H}+\mathrm{Al}=1,6 \mathrm{mmol}_{\mathrm{c}} \mathrm{dm}^{-3}$; $\mathrm{Cu}=0,07 ; \mathrm{Fe}=0,6 ; \mathrm{Mn}=0,3$ e $\mathrm{Zn}=0,6 \mathrm{mg} \mathrm{dm}^{-3}$.

As espécies de fungos micorrízicos empregadas neste estudo foram obtidas da mi coteca da Seção de Microbiologia do Solo do Departamento de Solos e Nutrição de Plantas da ESALQ, provenientes de vasos de multiplicação com milho, sendo elas: Acaulospora appendicula Spain (Sieverding \& Schenck), Acaul ospora morrowae(Spain \& Schenck), Scutellospora heterogama Nicol. \& Gerd. (Walker $\&$ Sanders), Gigaspora margarita (Becker \& Hall), Glomus macrocarpum (Tul \& Tul. var. macrocarpum) e Glomus etunicatum (Becker \& Gerdemann). Os esporos foram extraídos por peneiramento úmido (Gerdemann \& Nicolson, 1963) de uma alíquota do substrato e retidos em peneiras de malha variando de 50 a $250 \mu \mathrm{m}$, dependendo da espécie, após centrifugação em solução de sacarose a $40 \%$ (p/v), por 4 minutos, e lavados em água estéril. Para a remoção de fragmentos de hifas e outras impurezas, os esporos foram transferidos para frascos Erlenmeyer de $250 \mathrm{~mL}$ que continham $50 \mathrm{~g}$ desílica ( $>1$ e $<2 \mathrm{~mm}$ ) em $50 \mathrm{~mL}$ deágua eagitados, durante $30 \mathrm{~min}$, a $60 \mathrm{rpm}$. Para a recuperação dos esporos, repetiu-se o procedimento de peneiramento úmido após centrifugação em solução de sacarose.

Para eliminar ao máximo a influência de outros fatores biológicos no estudo, os esporos foram desinfestados à superfície com sol ução de hi poclorito de sódi o a 0,3 \% (v/v), por três mi nutos, com posterior lavagem em água destilada.

As doses de $\mathrm{Mn}$ foram adicionadas à areia lavada, na forma de $\mathrm{MnCl}_{2} \cdot 4 \mathrm{H}_{2} \mathrm{O}$ em solução, com vistas em obter 15, 30 e $75 \mathrm{mg} \mathrm{kg}^{-1}$ de $\mathrm{Mn}$, além de um controle sem adição de $\mathrm{Mn}$. Cada tratamento, em três repetições, foi acondicionado em placas de Petri com capacidade para $100 \mathrm{~g}$ do substrato. Com o auxílio de um mi croscópio ester eoscópio, os esporos de cada espécie foram transferidos em grupos de 20 para membranas de filtragem, marca Sartorius, de $47 \mathrm{~mm}$ de diâmetro, quadriculadas $(3 \times 3 \mathrm{~mm})$ e $3 \mu \mathrm{m}$ de porosidade. As membranas foram dobradas na forma de envel ope e introduzidas entre duas camadas de areia tratada com as doses de $\mathrm{Mn}$. A umidade da areia foi mantida em torno de $80 \%$ da capacidade máxima de retenção de água, pela adição de $20 \mathrm{~mL}$ da solução nutritiva de Steinberg (Foy et al., 1967), com 1/3 da concentração de $\mathrm{P}$, tamponada a pH 5 e isenta de $\mathrm{Mn}$. As placas foram embrulhadas em papel alumínio e incubadas, por 30 dias, em estufa biológica com temperatura controlada a $30 \stackrel{9}{ } \mathrm{C}$. Após esse período, os envelopes foram removidos e submetidos à avaliação da germinação dos esporos sob microscópio estereoscópio, no aumento de 45 a 90 vezes. Foram considerados germinados os esporos que apresentaram emergência do tubo germinativo.

Os resultados obtidos, em percentagem, foram submetidos à análise de variância com aplicação do teste $F$, em esquema fatorial $4 \times 6$, com prévia transformação dos dados para arcsen $(x / 100)^{1 / 2}$, em que x é a percentagem de germinação. Constatada a interação entre os fatores doses de $\mathrm{Mn}$ e fungos micorrízicos, aplicou-se o teste de Tukey $(P<0,05)$, para comparar os fungos mi corrízicos em cada dose de $\mathrm{Mn}$, bem como a análise de regressão, para avaliar o efeito das doses de $\mathrm{Mn}$ em cada espécie de fungo micorrízico. A variação percentual média da germinação dos esporos nas diferentes doses de $M n$, em relação ao tratamento sem $\mathrm{Mn}$, foi calculada segundo Lambais \& Cardoso (1989).

\section{RESULTADOS E DISCUSSÃO}

O resultado da análise estatística revelou interação entre os fatores avaliados a $P<0,01$, ou seja, o efeito das doses de $\mathrm{Mn}$ foi diferenciado para cada espécie de fungo micorrízico em estudo. Cada espécie de FMA apresentou maior ou menor sensibilidade à adição de $\mathrm{Mn}$ (Figuras 1 e 2). Uma delas, S. heterogama, tevesua germinação estimulada, até certo limite, pela adição de $\mathrm{Mn}$. As demais espécies apresentaram inibição da germinação dos esporos com o aumento da disponibilidade de $\mathrm{Mn}$ (Figura 1).

As espécies do gênero Glomus foram total mente inibidas na maior dose de $\mathrm{Mn}$. G. etunicatum foi a espécie que apresentou os menores índices de germinação, cerca de $20 \%$, mesmo no tratamento sem adição de Mn (Figura 2). Essa observação pode refletir a falta de adaptação dessa espécie ao substrato utilizado, o qual, quando acrescido de $\mathrm{Mn}$, limitou ainda mais a germinação dos esporos. G. margarita foi a espécie que apresentou o maior índice de germinação dos esporos e a que menos sofreu a interferência negativa da adição do $\mathrm{Mn}$. Lambais \& Cardoso (1989) relataram que doses crescentes de $\mathrm{Al}$ até $130 \mu \mathrm{mol} \mathrm{L}^{-1}$ não diminuíram significativamente a germinação dos esporos dessa espécie, em experimento semelhante. Alta resistência de G. margarita a metais também foi observada por Siqueira et al. (1985). Entretanto, Bartolome-E steban \& Schenck (1994) também observaram baixa sensibilidade de G. margarita em solos com crescentes saturações por Al. Por outro lado, Acaulospora appendicula foi a espécie que apresentou um dos maiores índices de germinação dos esporos no tratamento sem Mn (Figura 1), mas foi a que apresentou a maior inibição com a adição de $\mathrm{Mn}$, de forma semel hante ao tratamento com G. etunicatum (Figuras 1 e 2). 


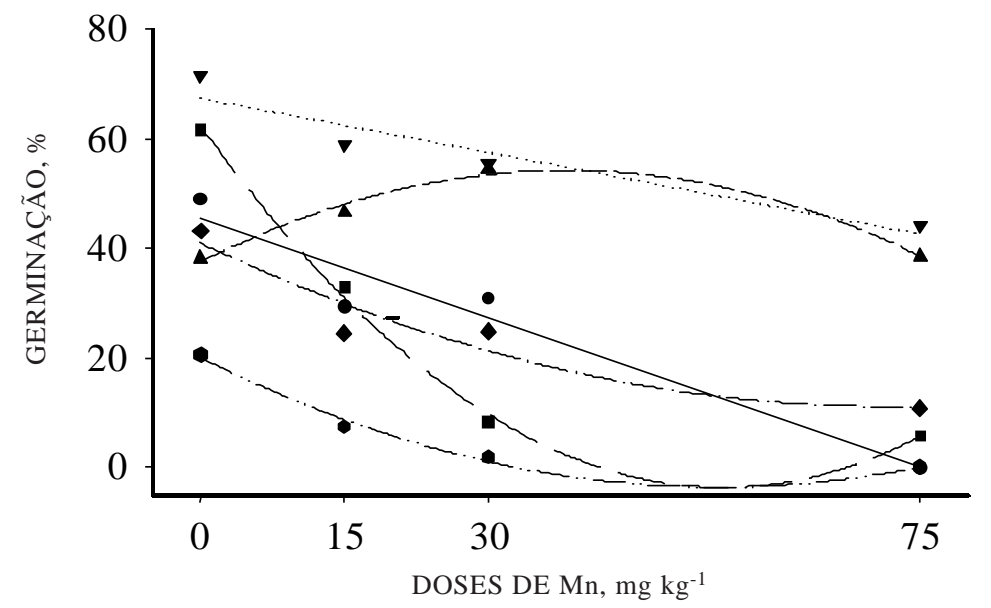

\footnotetext{
$\longrightarrow$ A. morrowae $\mathrm{Y}=45,5-0,61 \times\left(\mathrm{r}^{2}=0,94\right)$

- . G. margarita $\mathrm{Y}=67,4-0,33 \times\left(\mathrm{r}^{2}=0,90\right)$

- A. appendicula $\mathrm{Y}=62,4-2,42 \mathrm{x}+0,02 \mathrm{x}^{2}\left(\mathrm{r}^{2}=0,99\right)$

- G. macrocarpum $\mathrm{Y}=41-0,83 \mathrm{x}+0,006 \mathrm{x}^{2}\left(\mathrm{r}^{2}=0,91\right)$

- S. heterogama $\mathrm{Y}=37,56+0,87 \mathrm{x}-0,01 \mathrm{x}^{2}\left(\mathrm{r}^{2}=0,98\right) \longrightarrow$

- G. etunicatum $\mathrm{Y}=20,1-0,88 \mathrm{x}+0,008 \mathrm{x}^{2}\left(\mathrm{r}^{2}=0,99\right)$
}

Figura 1. Germinação de esporos de seis espécies de fungos micorrízicos arbusculares, de acordo com doses de Mn adicionadas ao substrato. Barras verticais indicam DMS em cada dose de Mn; coeficientes das equações são significativos a $P<0,05$.

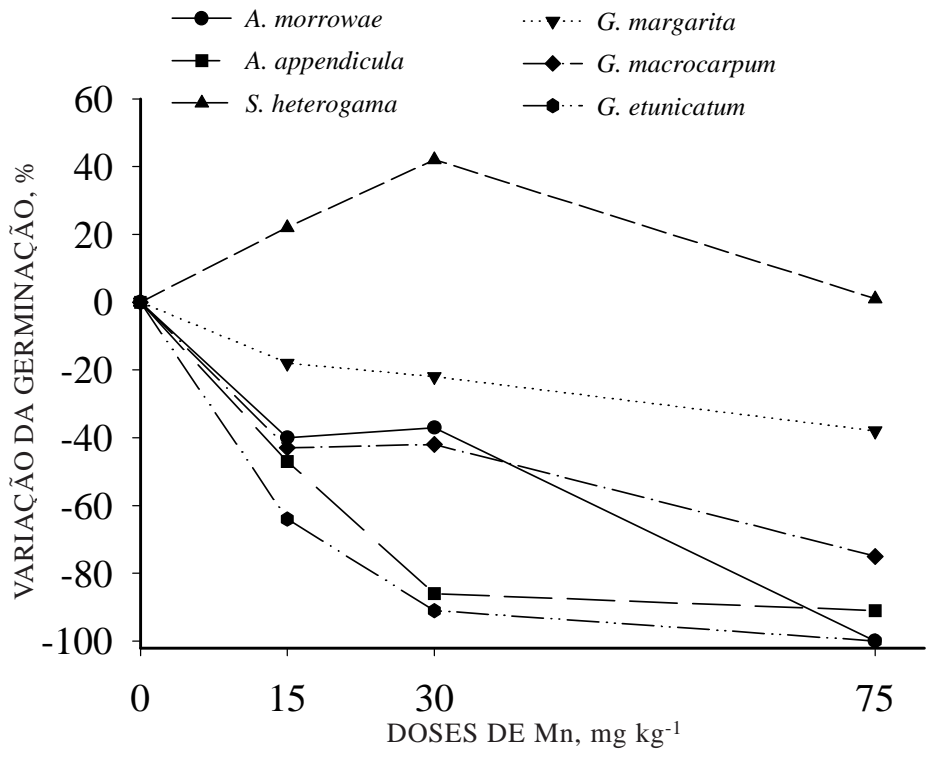

Fi gura 2. Variação percentual da germinação dos esporos de fungos mi corrízicos ar busculares de acordo com doses de Mn adicionadas ao substrato, em relação ao tratamento sem adição de Mn.

Considerando o efeito do $\mathrm{Mn}$, houve diminuição da germinação relativa dos esporos com o aumento das doses de $\mathrm{Mn}$ no meio de cultivo, com perdas do potencial germinativo médio de 32 , 49 e $74 \%$, nas doses de 15, 30 e $75 \mathrm{mg} \mathrm{kg}^{-1}$ deM ${ }^{2+}$, respectivamente, em comparação com o controle sem sua adição, considerado como $0 \%$ (Figura 2).

Deve-se considerar, entretanto, que a espécie S. heterogama, de modo contrárioao queocorreu com as demais espécies, teve sua germinação estimulada com o aumento das doses de $\mathrm{Mn}$ até $30 \mathrm{mg} \mathrm{kg}^{-1}$ (Figura 2), com variação $40 \%$ superior à do controle sem adição de $\mathrm{Mn}$. Na dosede $75 \mathrm{mg} \mathrm{kg}^{-1}$ de $\mathrm{Mn}$, não houve estímulo nem inibição da germinação dos esporos dessa espécie em comparação ao controlesem Mn. Tanto S. heterogama quanto G. margarita são citadas por Green et al. (1976) como espécies amplamente disseminadas em sol os áci dos, situação que favorece a disponibilidade de íons metálicos, como o Mn. O fato de G. margarita ter apresentado 
os menores decréscimos na germinação dos seus esporos com o aumento da disponibilidade de $\mathrm{Mn}$, além do estímulo no caso de S. heterogama, poderia estar relacionado com a prévia adaptação dessas espécies a ambientes com alta disponibilidade de $M n$ ou mesmo Al, já que a tolerância a metais por FMA parecenão ser específica (Weissenhorn et al., 1994).

Por outrolado, as espécies do gênero Glomus são relatadas como altamente susceptíveis a íons metálicos (Siqueira et al., 1984; Bartolome-E steban \& Schenck, 1994), e, juntamente com as do gênero Acaul ospora, foram as que mais sofreram os efeitos negativos da adição de Mn (Figura 2).

Lambais \& Cardoso (1989) observaram, em condições semel hantes, al ta ini bição da germi nação de esporos de G. macrocarpum em resposta ao aumento da disponibilidade de Al, corroborando os resultados obtidos por Siqueira et al. (1985), que constataram efeito inibitório ao G. mosseae em concentração de $1,5 \mathrm{mg} \mathrm{kg}^{-1}$ de Al, enquanto, para G. margarita, esse efeito somente ocorreu em concentrações superiores a $30 \mathrm{mg} \mathrm{kg}^{-1}$.

O conhecimento do comportamento de cada espécie ou isolado de FMA quanto à exposição ao Mn poderá ser utilizado para nortear trabal hos que pretendam utilizar essas espécies em ambientecom alta disponibilidade desse el emento, como nos casos de experimentos em que se faz necessária a autoclavagem do solo, procedimento que, dependendo do solo, leva a grande aumento da disponibilidade de $\mathrm{Mn}$. I sso não significa que as espécies menos susceptíveis à alta disponibilidade de $\mathrm{Mn}$ em estudos in vitro também sejam eficientes no estabelecimento da simbiose e na promoção de efeitos positivos em seus hospedeiros (Lambais \& Cardoso, 1988), sendo necessários estudos, em cada situação, com vistas em obter essas informações.

\section{CONCLUSÕES}

1. A germinação de esporos de fungos micorrízicos arbusculares mostrou-se diferencialmente prejudicada entre espécies e doses de $\mathrm{Mn}^{2+}$.

2. A germinação de esporos de $S$. heterogama foi estimulada por doses intermediárias de $\mathrm{Mn}^{2+}$ e não foi influenciada na dose de $75 \mathrm{mg} \mathrm{kg}^{-1}$, em relação ao controle sem adição de $\mathrm{Mn}^{2+}$.

3. As espécies dos gêneros Glomus e Acaul ospora mostraram-se mais sensíveis à presença de doses de $\mathrm{Mn}^{2+}$ em relação ao gênero Gigaspora.

\section{LITE RATURA CITADA}

BARTOLOME-ESTEBAN, H. \& SCHENCK, N.C. Spore germination and hyphal growth of arbuscular mycorrhizal fungi in relation to soil aluminum saturation. Mycologia, 86:217-226, 1994.
BOWEN, G.D. Mycorrhizal roles in tropical plants and ecosystems. In: MIKOLA, P., ed. Tropical mycorrhiza research. Oxford, Oxford University Press, 1980. p.165190.

CLARK, R.B. Arbuscular mycorrhizal adaptation, spore germination, root colonization, and host plant growth and mineral acquisition at low pH. Plant Soil, 192:15-22, 1997.

DAFT, M.J . \& EL-GIAHMI,A.A. Effects of Glomus infections on threelegumes. In: SANDERS, F.E.; MOSSE, B. \& TINKER, P.B., eds. Endomycorrhizas. London, Academic Press, 1975. p.470-484.

FOY, C.D. Physiological effects of hydrogen, aluminum and manganese toxicities in acid soil. In: ADAMS, F., ed. Soil acidity and liming. Madison, American Society of Agronomy, 1984. p.57-97.

FOY, C.D.; FLEMING, A.L; BURNS, G.R. \& ARMIGER, W.H. Characterization of differential aluminum among varieties of wheat and barley. Soil Sci. Soc. Am. J., 31:513-521, 1967.

GERDEMANN,J .N. \& NICHOLSON,T.H. Spores of mycorrhizal endogone species extracted from soil by wet sieving and decanting. Trans. Br. Mycol. Soc., 46:235-244, 1963.

GREEN, N.E.; GRAHAM, S.O. \& SCHENCK, N.C. The influence of $\mathrm{pH}$ on the germination of vesicular-arbuscular mycorrhizal spores. Mycologia, 68:929-934, 1976.

KOOMEN, I.; McGRATH, S.P. \& GILLER, I. Mycorrhizal infection of clover is delayed in soils contaminated with heavy metals from past sewage sludge applications. Soil Biol. Biochem., 22:871-873, 1990.

LAMBAIS, M.R. \& CARDOSO, E.J.B.N. Avaliação da germinação de esporos de fungos micorrízicos vesículo-arbusculares e da colonização micorrízica de Stylosanthes guianensis em solo ácido e distrófico. R. Bras. Ci. Solo, 12:249-255, 1988.

LAMBAIS, M.R. \& CARDOSO, E.J.B.N. Germinação de esporos e crescimento do tubo germinativo de fungos micorrízicos vesículo-arbusculares em diferentes concentrações de alumínio. R. Bras. Ci. Solo, 13:151-154, 1989.

SIQUEIRA, J .O.; HUBBELL, D.H. \& MAHMUD, A.W. Effect of liming on spore germination, germ tube growth and root colonization by vesicular-arbuscular mycorrhizal fungi. Plant Soil, 76:115-124, 1984.

SIQUEIRA, J .O.; SYLVIA, D.M.; GIBSON, J \& \& HUBBELL, D.H . Spores germination and germ tube growth of vesiculararbuscular mycorrhizal fungi. Can. J. Bot., 31:965-972, 1985.

WEISSENHORN, I.; GLASHOFF, A.; LEYVAL, C. \& BERTHELIN, J. Differential tolerance to $\mathrm{Cd}$ an $\mathrm{Zn}$ of arbuscular mycorrhizal (AM) fungal spores isolated from heavy metal-polluted and unpolluted soils. Plant Soil, 167:189-196, 1994.

WEISSENHORN, I.; LEYVAL, C. \& BERTHELIN, J . Cd-tolerant arbuscular mycorrhizal (AM) fungi from heavy-metal polluted soils. Plant Soil, 157:247-256, 1993. 
E.J .B.N. CARDOSO et al. 